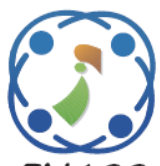

\title{
Performance Analysis for Hybrid Massive MIMO FSO/RF Links Based on Efficient Channel Codes
}

\author{
Tabarek A. Abdulabbas ${ }^{1 *}$ \\ Lwaa Faisal Abdulameer ${ }^{1}$ \\ ${ }^{I}$ Information and Communication Engineering Department, University of Baghdad, Iraq \\ * Corresponding author's Email: tabarak.abduladeem1203a@kecbu.uobaghdad.edu.iq
}

\begin{abstract}
FSO link suffers from a deep fade in some ranges of frequencies and it is very difficult to ensure the availability for this link especially in sever channel conditions such as dense fog, while RF link with mmWave working otherwise. FSO/RF hybrid system has emerged as a powerful solution for signal unavailability due to these conditions. The specific objective of this paper was to drive the error performance for the proposed coded hybrid communication system operating over atmospheric turbulence channel, considering gamma-gamma turbulence model for FSO and mmWave channel for RF. Error correction coding such as LT code, Polar code and Raptor code were used over FSO/RF link to mitigate turbulence fading and multipath effects. The numerical results of this paper show that the hybrid FSO/RF MIMO system implementing raptor code has a significant improvement over the system implementing LT or polar codes, $16 \times 16$ MIMO system implementing raptor code has a gain of $5 \mathrm{~dB}$ SNR when compared with the same system without implementing code. Additionally, the $16 \times 16$ MIMO hybrid system implemented Raptor code required $6 \mathrm{~dB}$ SNR to obtain BER of $10^{-3}$, while the $8 \times 8$ and $4 \times 4$ MIMO system with the same code required $8 \mathrm{~dB}$ and $9 \mathrm{~dB}$ SNR to achieve the same BER.
\end{abstract}

Keywords: FSO, RF, Massive MIMO, Polar code.

\section{Introduction}

The need for more advances in wireless networks to provide wide coverage area, gave us the demand for new techniques to take in advance. One of these techniques, which is more efficient, is free-space optical (FSO) communication [1].

FSO system has many features that gave it the upper hand to be used in $5^{\text {th }}$ Generation communication technology. Nevertheless, FSO system has difficulties too, for its use of the free space channel, which can make a regression in the signal transmitted through it. Moreover, there are more significant difficulties in the FSO system, which are atmospheric turbulence and lack of vision caused by the existence of air particles, heavy snowfall, and fog. On top of that, phenomena like severe winds and weak earthquakes can shake the towers that the transmitters and receivers are installed on and that can cause a pointing error [2]. We can obtain data rates equal to data rates achieved with FSO system by using Millimeter-wave Radio Frequency (RF) system. While Weather condition and atmospheric turbulence have an impact on both systems, it is not the same. For example, heavy rain can affect RF link performance while it has less effect on FSO link. Contrarily, Fog and atmospheric turbulence may make a regression in the behaviour of FSO link, but that will not be the case with RF link because it is not sensitive to fog and atmospheric turbulence [3].

For an FSO/RF system, each link is sensitive to different weather conditions. So, there will be a degradation in the signal only if both fog and rain occurred but that rarely happened [4].

In commerce, RF link used as a standby backup for the FSO link in order to obtain a hybrid solution, so that when the FSO channel is out of service, the RF link operates [5, 6]. A coding scheme for the hybrid channel has been presented by Vangala and Pishro-Nik [7, 8]. This scheme is based on nonuniform punctured Low-Density Parity Check (LDPC) codes. It depends on knowing the channel conditions at the transmitter immediately to specify 
the suitable modification in the code rates for FSO and RF transmission [4].

Lately, a paper suggests a hybrid FSO/RF schemes based on rateless channel codes for traditional communication channel with single transmitting antenna and single receiving antenna [4]. However, the work does not take into consideration improving the BER due to severe channel conditions for FSO link as well as millimeter region. This paper has investigated the use of massive MIMO technique which enhance the overall system performance.

Investigating the hybrid RF/FSO system with traditional Multiple Input Multiple Output (MIMO) scheme has been presented by a number of research works $[9,10]$. One of these works suggests a mixed RF and MIMO free-space optical (FSO) system based on a variable-gain dual-hop relay transmission scheme [9]. A dual-hop mixed FSO/RF system with a variable-gain Amplify-and-Forward (AF) relay was proposed, where the transmit diversity technique and the selection combining technique were adopted at the source and destination nodes, respectively. Moreover, RF and FSO links were characterized by Rayleigh distribution and M-distributed fading, respectively. Some other works $[11,12]$ examined the multi-diversity combining and selection schemes for the relay assisted mixed FSO/RF system with RF and FSO links being counted by Rayleigh and Gamma-Gamma fading channels, respectively [9].

The different structures used in the above works have been done with traditional MIMO and do not deal with the channel codes. In the present work, different channel codes for the proposed system are compared to distinguish the most suitable one to combat different channel conditions.

In this paper, we propose an investigation of the hybrid massive MIMO FSO/RF links using different channel codes (Luby Transform (LT) code, Polar code and Raptor code) working simultaneously. FSO is the dominant link when the weather conditions are suitable for FSO channel, while RF link with millimeter wave working otherwise. The main objective for this investigation is to provide coverages for everyone everywhere with the sever conditions affecting the wireless channel. This paper considers Minimum Mean Square Error (MMSE) and Zero Forcing (ZF) estimators to estimate the mmWave and Gamma-Gamma channel parameters that exploits the poor scattering nature of the channel.

The remaining sections of the paper are organized as follows; Section 2 presents the mathematical model of the two links (RF and FSO) for the proposed hybrid system, section 3 shows the results of this paper with a suitable explanation and discussion. Finally, section 4 presents the conclusion.

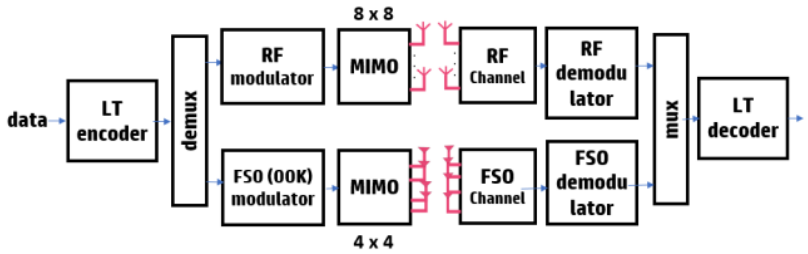

Figure. 1 The hybrid system block diagram

\section{Mathematical model}

The hybrid system consists of two links one for FSO and the other for RF. As shown in Fig. 1, both links have the same encoder, which is fed with a source data $S_{\mathrm{k}}$.

These data are encoded with a single encoder; The LT code is used to encode the information data. This code has been chose because of its ability of compacting fading effect [13] and in turn avoiding the loss of information [14, 15], especially due to scintillation and bad weather conditions, which tend to fluctuate the received signals. Moreover, it is simple in the mathematical model when compared to the Raptor code. Also, for the same reason, an $8 \times 8$ is used in the analysis of mathematical model for RF link and a $4 x 4$ for FSO link.

In Eq. (1), the entire data generated (u) was chosen with size of $\mathrm{k}$ bits,

$$
u=\left(u_{1}, u_{2}, \ldots \ldots . u_{k}\right)
$$

The LT code generates limitless output stream (x), by randomly choose degree (d) from a degree distribution $\rho(d)$,then performs bitwise XOR of the (d) source symbol chosen above. The encoder output:

$$
x=u_{i 1} \oplus u_{i 2} \ldots \ldots . \oplus u_{i k}
$$

The output symbols are generated step by step depending on repeating the above procedure.

\subsection{FSO link analysis}

Let us consider an ON-OFF Keying (OOK) to be used as a modulation scheme, where the transmitted signal $X_{F S O}(t) \in\{0,1\}$. However, there is no optical signal intensity during the period interval zero, and there is an optical signal during the period interval one. i.e.

$$
\begin{aligned}
& X_{F S O ~ 0}(t)=0, \quad 0 \leq t \leq T_{S} \\
& X_{F S O} 1(t)=1, \quad 0 \leq t \leq T_{S}
\end{aligned}
$$

The data information bits with period of symbol interval $\mathrm{T}_{\mathrm{s}}$ that are modulated by a light emitted diode (LED) for $\mathrm{m}^{\text {th }}$ emitter described at the output of 
transmitter as:

$$
X_{F S O}(t)=2 P_{T} \quad \sum_{k=0}^{T S} X_{F S O}(k)
$$

Where $\mathrm{P}_{\mathrm{T}}$ is the average power transmitted by LED.

The driving circuit in the block diagram is used to convert the information voltage signal coming from OOK modulator into a modulation current suitable for a LED source.

Now, let us consider the power is transmitted with 4 LEDs and detected with 4 Photodetectors (PDs). The Space Time Block Code (STBC) for a group of 4 symbols $\left(S_{1}, S_{2}, S_{3}\right.$ and $\left.S_{4}\right)$ that are transmitted by $\mathrm{M}=4$ antennas, during the used channel periods where $T_{s}=4$ is expressed below.

$$
S=\left[\begin{array}{cccc}
S_{1} & S_{2} & S_{3} & S_{4} \\
S_{2} & -S_{1}^{*} & S_{4}^{*} & -S_{3}^{*} \\
S_{3} & -S_{4} & -S_{1} & S_{2} \\
S_{4} & S_{3}^{*} & -S_{2} & -S_{1}^{*}
\end{array}\right]
$$

In Eq. (6), each row refers to spaces where information sends from different antennas and each column represents the time slot where each symbol is transmitted from a particular antenna at time $\mathrm{T}_{\mathrm{s}}(*$ in matrix represents the complex conjugate).

The design of the transmitted signals for different time slots from different LEDs is shown in Table 1.

These signals are transmitted via a wireless channel with channel gain in the form:

$$
H=\left[\begin{array}{llll}
h_{11} & h_{12} & h_{13} & h_{14} \\
h_{21} & h_{22} & h_{23} & h_{24} \\
h_{31} & h_{32} & h_{33} & h_{34} \\
h_{41} & h_{42} & h_{43} & h_{44}
\end{array}\right]
$$

Where, $h_{i j}$ is the channel coefficient between the $\mathrm{j}^{\text {th }}$ PD and $i^{\text {th }}$ LED.

Let us assume the two-sided Power Spectral Density (PSD) for an Additive White Gaussian Noise (AWGN) with zero mean and variance expressed as background noise and has the form:

Table 1. The design of the transmitted signals for different time slots from different LEDs

\begin{tabular}{|c|c|c|c|c|}
\hline Time interval & $\begin{array}{c}\text { Signal } \\
\text { transmit- } \\
\text { ed from } \\
\text { LED1 }\end{array}$ & $\begin{array}{c}\text { Signal } \\
\text { transmitted } \\
\text { from LED2 } 2\end{array}$ & $\begin{array}{c}\text { Signal } \\
\text { transmitted } \\
\text { from LED3 } 3\end{array}$ & $\begin{array}{c}\text { Signal } \\
\text { transmitted } \\
\text { from LED4 }\end{array}$ \\
\hline $0 \leq t \leq T_{S}$ & $X_{F S O} S_{1}$ & $X_{F S O} S_{2}$ & $X_{F S O} S_{3}$ & $X_{F S O} S_{4}$ \\
\hline$T_{s} \leq t \leq 2 T_{S}$ & $X_{F S O} S_{2}$ & $X_{F S O}\left(-S_{1}^{*}\right)$ & $X_{F S O} S_{4}^{*}$ & $X_{F S O}\left(-S_{3}^{*}\right)$ \\
\hline $2 T_{s} \leq t \leq 3 T_{S}$ & $X_{F S O} S_{3}$ & $X_{F S O}\left(-S_{4}\right)$ & $X_{F S O}\left(-S_{1}\right)$ & $X_{F S O} S_{2}$ \\
\hline $3 T_{s} \leq t \leq 4 T_{S}$ & $X_{F S O} S_{4}$ & $X_{F S O} S_{3}^{*}$ & $X_{F S O}\left(-S_{2}\right)$ & $X_{F S O}\left(-S_{1}^{*}\right)$ \\
\hline
\end{tabular}

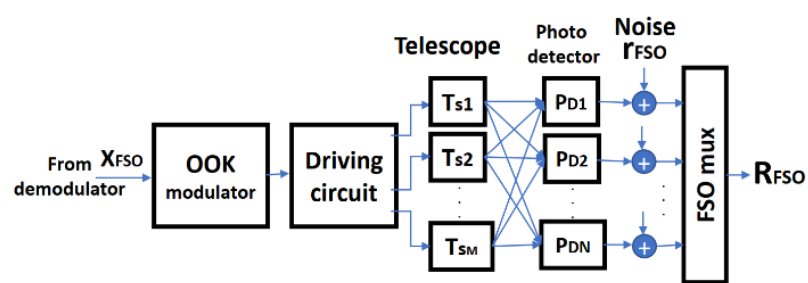

Figure. 2 Block diagram of FSO link with MIMO channel

Table 2. The form of output current from $\mathrm{PD}_{1}$

\begin{tabular}{|c|l|}
\hline Time interval & \multicolumn{1}{|c|}{$\mathrm{I}_{1}(\mathrm{t})$} \\
\hline $0 \leq t \leq T_{S}$ & $\begin{array}{l}2 P_{T}^{*} \mathrm{R}\left[h_{11} S_{1} X_{F S O}+h_{12} S_{2} X_{F S O}+\right. \\
\left.h_{13} S_{3} X_{F S O}+h_{14} S_{4} X_{F S O}+n_{1}\right]\end{array}$ \\
\hline$T_{s} \leq t \leq 2 T_{S}$ & $\begin{array}{l}2 P_{T}^{*} \mathrm{R}\left[h_{11} S_{2} X_{F S O}+h_{12}\left(-S_{1}{ }^{*}\right) X_{F S O}+\right. \\
\left.h_{13} S_{4}{ }^{*} X_{F S O}+h_{14}\left(-S_{3}{ }^{*}\right) X_{F S O}+n_{1}\right]\end{array}$ \\
\hline $2 T_{S} \leq t \leq 3 T_{S}$ & $\begin{array}{l}2 P_{T}^{*} \mathrm{R}\left[h_{11} S_{3} X_{F S O}+h_{12}\left(-S_{4}\right) X_{F S O}+\right. \\
\left.h_{13}\left(-S_{1}\right) X_{F S O}+h_{14} S_{2} X_{F S O}+n_{1}\right]\end{array}$ \\
\hline $3 T_{S} \leq t \leq 4 T_{S}$ & $\begin{array}{l}2 P_{T}^{*} \mathrm{R}\left[h_{11} S_{4} X_{F S O}+h_{12} S_{3}{ }^{*} X_{F S O}+\right. \\
\left.h_{13}\left(-S_{2}\right) X_{F S O}+h_{14}\left(-S_{1}{ }^{*}\right) X_{F S O}+n_{1}\right]\end{array}$ \\
\hline
\end{tabular}

$$
n=\frac{N o}{2}=2 I_{B} q
$$

Where $q$ is the electron charge and $I_{B}$ is the photocurrent.

The variance can be expressed in Eq. (9).

$$
\sigma_{n}^{2}=\frac{N o}{2 T s}=\frac{2 T_{B} q}{T s}
$$

The output current from $\mathrm{PD}_{1}$ has the forms in Table 2. $\left(\mathrm{PD}_{2}, \mathrm{PD}_{3}\right.$ and $\left.\mathrm{PD}_{4}\right)$ for different intervals 0 $\leq \mathrm{t} \leq 4 \mathrm{~T}_{\mathrm{s}}$ are expressed with the same scenario in Table 2 according to Table 1.

Where $\mathrm{R}$ is the photodetector responsivity.

In Table 2, the transmitted signal from $\mathrm{M}^{\text {th }}$ LED is multiplied by a channel gain $h_{j, i}$ at the $\mathrm{N}^{\text {th }} \mathrm{PD}$.

These output currents from photodetectors are correlated by a correlator at the receiver in the form:

$$
\begin{aligned}
& R_{1}=2 P_{T}^{2} \mathrm{R} \sum_{t=0}^{4 T_{S}} I_{1}(t) X_{F S O}= \\
& 2 P_{T}^{2} \mathrm{R}\left[\left(h_{11} S_{1}+h_{12} S_{2}+h_{13} S_{3}+h_{14} S_{4}\right)+\right. \\
& \left(h_{11} S_{2}+h_{12}\left(-S_{1}^{*}\right)+h_{13} S_{4}^{*}+h_{14}\left(-S_{3}^{*}\right)+\right. \\
& h_{11} S_{3}+h_{12}\left(-S_{4}\right)+h_{13}\left(-S_{1}\right)+h_{14} S_{2}+ \\
& h_{11} S_{4}+h_{12}\left(S_{3}^{*}\right)+h_{13}\left(-S_{2}\right)+ \\
& \left.h_{14}\left(-S_{1}^{*}\right)+N_{1}\right]
\end{aligned}
$$

Where $\sum_{t=0}^{4 T_{s}} n_{1} X_{F S O}=N_{1}$, represents the equivalent noise.

Similarly, we can get $\mathrm{R}_{2}, \mathrm{R}_{3}$ and $\mathrm{R}_{4}$.

These correlated signals are multiplied by the conjugate transpose of the channel gain to estimate the emitted bits, thus: 


$$
\left[\begin{array}{c}
D_{s 1} \\
D_{s 2} \\
D_{s 3} \\
D_{s 4}
\end{array}\right]=H^{*} R_{1}
$$

Where, $H^{*}$ is the conjugate transpose of the channel matrix.

However, the estimated bits are computed by taking the sign of the decision variable as follows: $S_{1}=\operatorname{Sign}\left(D_{s 1}\right), S_{2}=\operatorname{Sign}\left(D_{s 2}\right), S_{3}=\operatorname{Sign}\left(D_{s 3}\right)$ and $S_{4}=\operatorname{Sign}\left(D_{s 4}\right)$. The similar processes have been considered for the rest symbols.

\subsection{RF link analysis}

Let us consider a QPSK to be used as a modulation scheme for RF link. The transmitter maps $m_{R F}=\log _{2}(M)$ binary symbols to an M-ary PSK Quadrature Phase Shift Keying (QPSK) where M =2.

Recall that the data is regenerated with k-bits size. These data are encoded by multiplying the information bits by a generator matrix to produce unlimited output streams as mentioned earlier.

The block diagram of RF link can be described with Fig. 3:

Now, let us consider an mmWave massive MIMO scheme with an LT encoder. Let the transmitter equipped with 8 transmitted antenna (i.e. $X_{R F} \in \mathbb{C}^{M \times 1}$ ) and the receiver is equipped with 8 receiving antennas (i.e. $Y_{R F} \in \mathbb{C}^{N \times 1}$ ). So, the channel gain between $T_{X_{R F}}$ and $R_{X_{R F}}$ is $H_{R F} \in$ $\mathbb{C}^{N \times M}$.

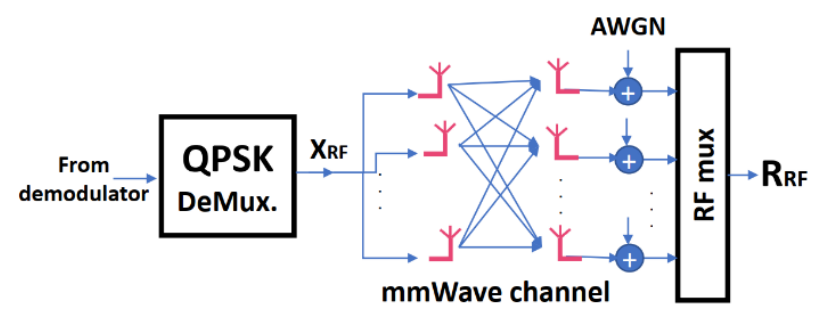

Figure. 3 RF link block diagram
The STBC for a group of 8 symbols $\left(S_{1}, S_{2}, S_{3}\right.$ $S_{4}, S_{5}, S_{6}, S_{7}$ and $S_{8}$ ) that are transmitted by $\mathrm{M}=8$ antennas, during $\mathrm{T}_{\mathrm{s}}=8$ is expressed in Eq. (12).

$$
\begin{aligned}
& S= \\
& {\left[\begin{array}{rrrrrrrl}
S_{1} & S_{2} & S_{3} & S_{4} & S_{5} & S_{6} & S_{7} & S_{8} \\
S_{2} & S_{1} & S_{4} & S_{3} & S_{6} & S_{5} & S_{8} & S_{7} \\
S_{3} & S_{2} & S_{1} & S_{4} & S_{7} & S_{6} & S_{5} & S_{8} \\
S_{4} & S_{3} & S_{2} & S_{1} & S_{8} & S_{7} & S_{6} & S_{5} \\
-S_{5}^{*} & -S_{8}^{*} & -S_{7}^{*} & -S_{6}^{*} & -S_{1}^{*} & -S_{4}^{*} & -S_{3}^{*} & -S_{2}^{*} \\
-S_{6}^{*} & -S_{5}^{*} & -S_{8}^{*} & -S_{7}^{*} & -S_{2}^{*} & -S_{1}^{*} & -S_{4}^{*} & -S_{3}^{*} \\
-S_{7}^{*} & -S_{6}^{*} & -S_{5}^{*} & -S_{8}^{*} & -S_{3}^{*} & -S_{2}^{*} & -S_{1}^{*} & -S_{4}^{*} \\
-S_{8}^{*} & -S_{7}^{*} & -S_{6}^{*} & -S_{5}^{*} & -S_{4}^{*} & -S_{3}^{*} & -S_{2}^{*} & -S_{1}^{*}
\end{array}\right]}
\end{aligned}
$$

However, to analyze the RF system, we need to calculate the Signal to Noise Ratio (SNR).

Towards this, we evaluate the beamforming to maximize the SNR. At the transmitter, the transmitted signals have been designed as in Table 3.

These signals are transmitted in space time scenario, simultaneously from the Base Station (BS) and received by N-antenna at Mobile Station (MS).

Consider the $8 \times 8$ channel gain for RF link is $G$ which is denoted by:

$$
G=\left[\begin{array}{ccc}
g_{11} & \cdots & g_{18} \\
\vdots & \ddots & \vdots \\
g_{81} & \cdots & g_{88}
\end{array}\right]
$$

Thus, the model of the received signals are denoted by:

$$
r_{R F}=G_{8 \times 8} X_{R F}+N_{R F}
$$

Where $r_{R F} \in \mathbb{C}^{N \times 1}, X_{R F} \in \mathbb{C}^{M \times 1}, N_{R F} \in \mathbb{C}^{M \times 1}$, $G \in \mathbb{C}^{N \times M}$

$$
r_{R F}=\left(r_{R F_{1}}, r_{R F_{2}}, \ldots \ldots \ldots, r_{R F_{k}}\right)^{T}
$$

Table 3. RF link's transmitted signals at different time slots

\begin{tabular}{|c|c|c|c|c|c|c|c|c|}
\hline Time & $A_{n} t_{1}$ & $A_{n} t_{2}$ & $A_{n} t_{3}$ & $A_{n} t_{4}$ & $A_{n} t_{5}$ & $A_{n} t_{6}$ & $A_{n} t_{7}$ & $A_{n} t_{8}$ \\
\hline $0 \leq t \leq T_{s}$ & $S_{1} X_{R F}$ & $S_{2} X_{R F}$ & $S_{3} X_{R F}$ & $S_{4} X_{R F}$ & $S_{5} X_{R F}$ & $S_{6} X_{R F}$ & $S_{7} X_{R F}$ & $S_{8} X_{R F}$ \\
\hline$T_{s} \leq t \leq 2 T_{s}$ & $S_{2} X_{R F}$ & $S_{1} X_{R F}$ & $S_{4} X_{R F}$ & $S_{3} X_{R F}$ & $S_{6} X_{R F}$ & $S_{5} X_{R F}$ & $S_{8} X_{R F}$ & $S_{7} X_{R F}$ \\
\hline $2 T_{s} \leq t \leq 3 T_{s}$ & $S_{3} X_{R F}$ & $S_{2} X_{R F}$ & $S_{1} X_{R F}$ & $S_{4} X_{R F}$ & $S_{7} X_{R F}$ & $S_{6} X_{R F}$ & $S_{5} X_{R F}$ & $S_{8} X_{R F}$ \\
\hline $3 T_{s} \leq t \leq 4 T_{S}$ & $S_{4} X_{R F}$ & $S_{3} X_{R F}$ & $S_{2} X_{R F}$ & $S_{1} X_{R F}$ & $S_{8} X_{R F}$ & $S_{7} X_{R F}$ & $S_{6} X_{R F}$ & $S_{5} X_{R F}$ \\
\hline $4 T_{s} \leq t \leq 5 T_{s}$ & $-S_{5}^{*} X_{R F}$ & $-S_{8}^{*} X_{R F}$ & $-S_{7}^{*} X_{R F}$ & $-S_{6}^{*} X_{R F}$ & $-S_{1}^{*} X_{R F}$ & $-S_{4}^{*} X_{R F}$ & $-S_{3}^{*} X_{R F}$ & $-S_{2}^{*} X_{R F}$ \\
\hline $5 T_{s} \leq t \leq 6 T_{S}$ & $-S_{6}^{*} X_{R F}$ & $-S_{5}^{*} X_{R F}$ & $-S_{8}^{*} X_{R F}$ & $-S_{7}^{*} X_{R F}$ & $-S_{2}^{*} X_{R F}$ & $-S_{1}^{*} X_{R F}$ & $-S_{4}^{*} X_{R F}$ & $-S_{3}^{*} X_{R F}$ \\
\hline $6 T_{s} \leq t \leq 7 T_{s}$ & $-S_{7}^{*} X_{R F}$ & $-S_{6}^{*} X_{R F}$ & $-S_{5}^{*} X_{R F}$ & $-S_{8}^{*} X_{R F}$ & $-S_{3}^{*} X_{R F}$ & $-S_{2}^{*} X_{R F}$ & $-S_{1}^{*} X_{R F}$ & $-S_{4}^{*} X_{R F}$ \\
\hline $7 T_{s} \leq t \leq 8 T_{S}$ & $-S_{8}^{*} X_{R F}$ & $-S_{7}^{*} X_{R F}$ & $-S_{6}^{*} X_{R F}$ & $-S_{5}^{*} X_{R F}$ & $-S_{4}^{*} X_{R F}$ & $-S_{3}^{*} X_{R F}$ & $-S_{2}^{*} X_{R F}$ & $-S_{1}^{*} X_{R F}$ \\
\hline
\end{tabular}




$$
N_{R F}=\left(N_{R F_{1}}, N_{R F_{2}}, \ldots \ldots \ldots, N_{R F_{k}}\right)^{T}
$$

Where $N_{R F}$ represents the equivalent noise at the receiver.

However, to look at the model of the received signals for different space-time coding techniques, we will consider the received signals by the $1^{\text {st }}$ received antenna $\left(R_{x_{1}}\right)$ which are as in Table 4 . The same scenario in Table 4 will go on the received signals for different antennas $\left(R_{x_{2}}, R_{x_{3}}, R_{x_{4}}, R_{x_{5}}\right.$, $\left.R_{x_{6}}, R_{x_{7}}, R_{x_{8}}\right)$ and different intervals $0 \leq \mathrm{t} \leq 8 \mathrm{~T}_{\mathrm{s}}$ according to Table 3 .

However, these signals at the antennas of the RF link are analyzed as follow:

First, Recall Eq. (13) which is the model of the received signal for the wireless communications.

Now, consider the expected value of AWGN, which is the noise power provided at each receiver,

Table 4 . The received signals by the $1^{\text {st }}$ received antenna $\left(R_{x_{1}}\right)$

\begin{tabular}{|c|c|}
\hline Time interval & Received signals at $R_{x_{1}}$ \\
\hline $0 \leq t \leq T_{s}$ & $\begin{array}{l}\left(g_{11} S_{1} X_{R F}+g_{12} S_{2} X_{R F}+g_{13} S_{3} X_{R F}+\right. \\
g_{14} S_{4} X_{R F}+g_{15} S_{5} X_{R F}+g_{16} S_{6} X_{R F}+ \\
\left.g_{17} S_{7} X_{R F}+g_{18} S_{8} X_{R F}+N_{R F_{1}}\right)\end{array}$ \\
\hline$T_{s} \leq t \leq 2 T_{s}$ & $\begin{array}{l}\left(g_{11} S_{2} X_{R F}+g_{12} S_{1} X_{R F}+g_{13} S_{4} X_{R F}+\right. \\
g_{14} S_{3} X_{R F}+g_{15} S_{6} X_{R F}+g_{16} S_{5} X_{R F}+ \\
\left.g_{17} S_{8} X_{R F}+g_{18} S_{7} X_{R F}+N_{R F_{2}}\right)\end{array}$ \\
\hline $\begin{array}{l}2 T_{s} \leq t \\
\leq 3 T_{s}\end{array}$ & $\begin{array}{l}\left(g_{11} S_{3} X_{R F}+g_{12} S_{1} X_{R F}+g_{13} S_{2} X_{R F}+\right. \\
g_{14} S_{4} X_{R F}+g_{15} S_{7} X_{R F}+g_{16} S_{6} X_{R F}+ \\
\left.g_{17} S_{5} X_{R F}+g_{18} S_{8} X_{R F}+N_{R F_{3}}\right) \\
\end{array}$ \\
\hline $\begin{array}{l}3 T_{s} \leq t \\
\leq 4 T_{s}\end{array}$ & $\begin{array}{l}\left(g_{11} S_{1} X_{R F}+g_{12} S_{2} X_{R F}+g_{13} S_{3} X_{R F}+\right. \\
g_{14} S_{4} X_{R F}+g_{15} S_{5} X_{R F}+g_{16} S_{6} X_{R F}+ \\
\left.g_{17} S_{7} X_{R F}+g_{18} S_{8} X_{R F}+N_{R F_{4}}\right)\end{array}$ \\
\hline $\begin{array}{l}4 T_{s} \leq t \\
\leq 5 T_{s}\end{array}$ & $\begin{array}{l}\left(g_{11}\left(-S_{5}^{*}\right) X_{R F}+g_{12}\left(-S_{8}^{*}\right) X_{R F}+\right. \\
g_{13}\left(-S_{7}^{*}\right) X_{R F}+g_{14}\left(-S_{6}^{*}\right) X_{R F}+ \\
g_{15}\left(-S_{1}^{*}\right) X_{R F}+g_{16}\left(-S_{4}^{*}\right) X_{R F}+ \\
\left.g_{17}\left(-S_{3}^{*}\right) X_{R F}+g_{18}\left(-S_{2}^{*}\right) X_{R F}+N_{R F_{5}}\right)\end{array}$ \\
\hline $\begin{array}{l}5 T_{s} \leq t \\
\leq 6 T_{s}\end{array}$ & $\begin{array}{l}\left(g_{11}\left(-S_{6}^{*}\right) X_{R F}+g_{12}\left(-S_{5}^{*}\right) X_{R F}+\right. \\
g_{13}\left(-S_{8}^{*}\right) X_{R F}+g_{14}\left(-S_{7}^{*}\right) X_{R F}+ \\
g_{15}\left(-S_{2}^{*}\right) X_{R F}+g_{16}\left(-S_{1}^{*}\right) X_{R F}+ \\
\left.g_{17}\left(-S_{4}^{*}\right) X_{R F}+g_{18}\left(-S_{3}^{*}\right) X_{R F}+N_{R F_{6}}\right)\end{array}$ \\
\hline $\begin{array}{l}6 T_{s} \leq t \\
\leq 7 T_{s}\end{array}$ & $\begin{array}{l}\left(g_{11}\left(-S_{7}^{*}\right) X_{R F}+g_{12}\left(-S_{6}^{*}\right) X_{R F}+\right. \\
g_{13}\left(-S_{5}^{*}\right) X_{R F}+g_{14}\left(-S_{8}^{*}\right) X_{R F}+ \\
g_{15}\left(-S_{3}^{*}\right) X_{R F}+g_{16}\left(-S_{2}^{*}\right) X_{R F}+ \\
\left.g_{17}\left(-S_{1}^{*}\right) X_{R F}+g_{18}\left(-S_{4}^{*}\right) X_{R F}+N_{R F_{7}}\right)\end{array}$ \\
\hline $\begin{array}{l}7 T_{s} \leq t \\
\leq 8 T_{s}\end{array}$ & $\begin{array}{l}\left(g_{11}\left(-S_{8}^{*}\right) X_{R F}+g_{12}\left(-S_{7}^{*}\right) X_{R F}+\right. \\
g_{13}\left(-S_{6}^{*}\right) X_{R F}+g_{14}\left(-S_{5}^{*}\right) X_{R F}+ \\
g_{15}\left(-S_{4}^{*}\right) X_{R F}+g_{16}\left(-S_{3}^{*}\right) X_{R F}+ \\
\left.g_{17}\left(-S_{2}^{*}\right) X_{R F}+g_{18}\left(-S_{1}^{*}\right) X_{R F}+N_{R F_{8}}\right)\end{array}$ \\
\hline
\end{tabular}

is:

$$
E\left\{\left|N_{i}(k)\right|^{2}\right\}=\sigma_{N}^{2}
$$

All signals received at $\mathrm{N}$ antennas at the receiver side must be combined to detect the transmitted signal $X_{R F}$.

So, $\left(\bar{R}_{1}, \bar{R}_{2}, \ldots \ldots . \bar{R}_{n}\right)$ are combined at the output of all receivers.

The beamforming technique is used to combine the received signals for $\mathrm{L}$ paths, which provides maximizing in the SNR and improvement in the BER performance of the RF link at the mmWaves that suffer from sever fading due to multipath effects and degradation in the SNR specifically at these $\mathrm{mm}$ ranges of frequencies.

However, the combination of all signals is denoted by multiplying the beamformer (W) by the received signal $\bar{r}$, in other meaning has the form:

$$
\left[W_{1}^{*} r_{1}+W_{2}^{*} r_{2}+W_{3}^{*} r_{3} \ldots \ldots \ldots+W_{L}^{*} r_{N}\right]
$$

Where $\mathrm{L}$ is the number of paths arrived at the MS.

The analysis is achieved by considering that all columns in the matrix described in Eq. (12) are orthogonal and the channel state information (CSI) is available, the maximum ratio combiner is represented as

$$
\left[\begin{array}{llll}
W_{1}^{*} & W_{2}^{*} & \ldots & \ldots \\
W_{L}^{*}
\end{array}\right]\left[\begin{array}{c}
r_{1} \\
r_{2} \\
\vdots \\
r_{N}
\end{array}\right]=\bar{W}_{L}^{H} \bar{r}_{N}
$$

Where, the uppercase $(\mathrm{H})$ for $\mathrm{W}$ represents the Hermitian matrix for $\mathrm{W}$, which means the conjugate of a matrix for complex entries.

According to the above equations, the output of beamformer is:

$$
\bar{W}^{H}\left(\bar{G} X_{R F}+\bar{N}_{R F}\right)=\bar{W}^{H} \bar{G} X_{R F}+\bar{W}^{H} \bar{N}_{R F}
$$

The first term represents the signal components, while the second term represents the noise components and represents the vector.

The SNR at the output of beamformer is computed by dividing the signal power to the noise power.

So, the signal power is denoted by:

$$
\text { Signal power }=\left|\bar{W}^{H} \bar{G}\right|^{2} p
$$

The effective noise at the output of beamformer is denoted by: 
$\bar{N}_{R F} \bar{N}_{R F}{ }^{H}=\left[\begin{array}{c}N_{R F_{1}} \\ N_{R F_{2}} \\ \vdots \\ N_{R F_{N}}\end{array}\right]\left[\begin{array}{lllll}N_{R F_{1}} N_{R F_{2}} & \ldots & \ldots & N_{R F_{N}}\end{array}\right]=$

$\left[\begin{array}{cccc}\left|N_{R F_{1}}\right|^{2} & N_{R F_{1}} N_{R F_{2}} & \cdots & N_{R F_{1}} N_{R F_{N}}^{*} \\ N_{R F_{1}} N_{R F_{1}}^{*} & \left|N_{R F_{2}}\right|^{2} & \cdots & N_{R F_{2}} N_{R F_{N}}^{*} \\ \vdots & \ddots & & \left|N_{R F_{N}}\right|^{2}\end{array}\right]$

The expected variance of the covariance matrix is denoted by:

$E\left\{\left[\begin{array}{cccc}\left|N_{R F_{1}}\right|^{2} & N_{R F_{1}} N_{R F_{2}} & \cdots & N_{R F_{1}} N_{R F_{N}}^{*} \\ N_{R F_{1}} N_{R F_{1}}^{*} & \left|N_{R F_{2}}\right|^{2} & \cdots & N_{R F_{2}} N_{R F_{N}}^{*} \\ \vdots & \cdots & & \left|N_{R F_{N}}\right|^{2}\end{array}\right]\right\}$

Since, the expected value of the $N_{R F_{1}} N_{R F_{1}}^{*}$ is equal to zero and for $\left.\left|N_{R F}\right|_{i i}\right|^{2}$ is equal to $\sigma_{N_{R F}}^{2}$, then:

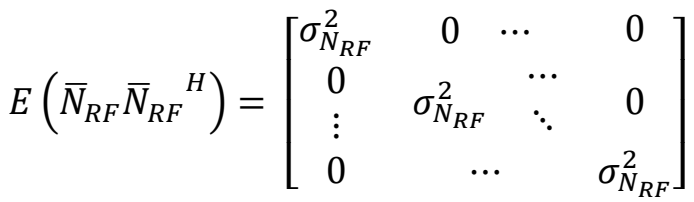

According to the process above the noise power is computed by:

$$
\begin{aligned}
& \text { Noice power }=\left(\bar{W}^{H} \bar{N}_{R F}\right)\left(\bar{W}^{H} \bar{N}_{R F}{ }^{*}\right)= \\
& \sigma_{N_{R F}}^{2}\|\bar{W}\|^{2}
\end{aligned}
$$

Which, represents the noise at the output of beamformer.

As a result, the maximized SNR at the output of the beamformer for the RF link is denoted by:

$$
S N R_{R F_{\text {maximized }}}=\frac{\left|\bar{W}^{H} \bar{G}\right|^{2} p}{\sigma_{N_{R F}}^{2} \bar{W}^{H} W}
$$

Remember that the RF link with single point to point $8 \times 8$ MIMO system is considered.

The Minimum Mean Square Error (MMSE) for single input single output (SISO) communication system is obtained by minimizing the Mean Square Error (MSE), which defines as $E\left\{\left\|\hat{X}_{R F}-X_{R F}\right\|^{2}\right\}$, where $\hat{X}_{R F}$ is the estimated signal. Let us consider the number of antennas at the transmitter is more than the number of antennas at the receiver (i.e. $\mathrm{M} \geq \mathrm{N}$ ).

$$
\text { Let } \hat{X}_{R F}=\bar{L}^{T} \bar{R}
$$

Then, the MSE is denoted by:

$$
\operatorname{MSE}\left(\hat{X}_{R F}\right)=E\left\{\left\|\bar{L}^{T} \bar{R}-X_{R F}\right\|^{2}\right\}
$$

Where $\mathrm{L}$ is the linear estimator and $\bar{R}$ is the received vector.

However, to minimize the above formula.

$$
\begin{aligned}
& \min E\left\{\left\|\bar{L}^{T} \bar{R}-X_{R F}\right\|^{2}\right\}=E\left\{\left(\bar{K}^{T} \bar{R}-\right.\right. \\
& \left.\left.X_{R F}\right)\left(\bar{K}^{T} \bar{R}-X_{R F}\right)^{T}\right\}=E\left\{\bar{L}^{T} \bar{R} \bar{R}^{T} \bar{K}-\right. \\
& \left.X_{R F} \bar{R}^{T} \bar{L}-\bar{L}^{T} \bar{R} X_{R F}{ }^{T}+X_{R F} X_{R F}^{T}\right\}
\end{aligned}
$$

Consider the covariance matrix of the received vector $\bar{R}$ is:

$$
E\left(\bar{R} \bar{R}^{T}\right)=\mathcal{R}_{R R}
$$

Where, $\mathcal{R}$ represents the covariance.

The cross covariance for transmitted and received vectors is:

$$
\begin{aligned}
& E\left(X_{R F} \bar{R}^{T}\right)=\mathcal{R}_{X_{R F} R} \\
& E\left(R \bar{X}_{R F}\right)=\mathcal{R}_{R X_{R F}}
\end{aligned}
$$

From the above relationships, we have:

$$
\begin{aligned}
& \min E\left\{\left\|\bar{L}^{T} \bar{R}-X_{R F}\right\|^{2}\right\}=\bar{L}^{T} \mathcal{R}_{R R} \bar{L}- \\
& \mathcal{R}_{X_{R F} R} \bar{L}-\bar{L}^{T} \mathcal{R}_{R X_{R F}}+\mathcal{R}_{X_{R F} X_{R F}}
\end{aligned}
$$

The minimized formula of the above equation is:

$$
\begin{aligned}
& \min \left\{\bar{L}^{T} \mathcal{R}_{R R} \bar{L}-\mathcal{R}_{X_{R F} R} \bar{L}-\bar{L}^{T} \mathcal{R}_{R X_{R F}}+\right. \\
& \left.\mathcal{R}_{X_{R F} X_{R F}}\right\}= \\
& \min \left\{\frac{\bar{L}^{T} \mathcal{R}_{R R} \bar{L}-2 \bar{L} \mathcal{R}_{R X_{R F}}+\mathcal{R}_{X_{R F} X_{R F}}}{f(\bar{L})}\right\}
\end{aligned}
$$

Where $f(\bar{L})=\frac{\partial f}{\partial \bar{L}}$

For minimum error:

$f(\bar{L})=0$, then:

$$
\begin{aligned}
& 2 \mathcal{R}_{R R} \bar{L}-2 \mathcal{R}_{R X_{R F}}=0 \\
& \mathcal{R}_{R R} \bar{L}=\mathcal{R}_{R X_{R F}} \\
& \bar{L}=\frac{\mathcal{R}_{R X_{R F}}}{\mathcal{R}_{R R}}=\mathcal{R}_{R R}^{-1} \mathcal{R}_{R X_{R F}}
\end{aligned}
$$

This is the Linear Minimum Mean Square Error 
(LMMSE) estimator.

However, the estimated signal is:

$$
\widehat{X}_{R F}=\bar{L}^{T} \bar{R}
$$

For the complex vector, the LMMSE denoted by:

$$
\begin{aligned}
& \hat{X}_{R F}=\bar{L}^{H} \bar{R} \\
& \hat{X}_{R F}=\mathcal{R}_{R R}{ }^{-1} \mathcal{R}_{R X_{R F}} \bar{R}
\end{aligned}
$$

Where $H$ here denotes the Hermitian matrix.

However, for $8 \times 8 \mathrm{MIMO}$, the exceeded value of the transmitted signal is:

$$
\mathcal{R}_{X_{R F} X_{R F}}=E\left\{\bar{X}_{R F} \bar{X}_{R F}^{H}\right\}
$$

Then the covariance of the transmitted symbols becomes:

$$
\begin{aligned}
& \mathcal{R}_{X_{R F} X_{R F}}=E\left\{\left[\begin{array}{c}
X_{R F_{1}} \\
X_{R F_{2}} \\
\vdots \\
X_{R F_{t}}
\end{array}\right]\left[\begin{array}{llll}
X_{R F_{1}}^{*} & X_{R F_{2}}^{*} & \cdots & X_{R F_{t}}^{*}
\end{array}\right]\right\} \\
& =E\left\{\left[\begin{array}{cccc}
\left|X_{R F_{1}}\right|^{2} & X_{R F_{1}} X_{R F_{2}}^{*} & \cdots & X_{R F_{1}} X_{R F_{t}}^{*} \\
X_{R F_{2}} X_{R F_{1}}^{*} & \left|X_{2}\right|^{2} & \cdots & X_{R F_{2}} X_{R F_{t}}^{*} \\
\vdots & \ddots & & \left|X_{R F_{t}}\right|^{2}
\end{array}\right]\right\} \\
& \mathcal{R}_{X_{R F} X_{R F}}=\left[\begin{array}{cccc}
P_{t} & 0 & \cdots & 0 \\
0 & P_{t} & \cdots & 0 \\
\vdots & & \ddots & \\
0 & \multicolumn{1}{c}{\cdots} & & P_{t}
\end{array}\right]=P_{t} I_{t}
\end{aligned}
$$

Where, $P_{t}$ is the transmitted power and $I_{t}$ is the unity matrix.

$$
\begin{aligned}
& \mathcal{R}_{R R}=E\left\{\bar{R}^{H} \bar{R}\right\}=E\left\{\left(G_{8 \times 8} \bar{X}_{R F}+\right.\right. \\
& \left.\left.n_{R F}\right)\left(G_{8 \times 8} X_{R F}+n_{R F}\right)^{H}\right\}= \\
& E\left\{\left(\bar{G}_{8 \times 8} \bar{X}_{R F} \bar{X}_{R F}{ }^{H} \bar{G}_{8 \times 8}{ }^{H}+\right.\right. \\
& n_{R F} \bar{X}_{R F}{ }^{H} \bar{G}_{8 \times 8}{ }^{H}+\bar{G}_{8 \times 8} X_{R F} \bar{n}_{R F}{ }^{H}+ \\
& \left.\left.n_{R F} n_{R F}{ }^{H}\right)\right\}
\end{aligned}
$$

The second and third terms in the above equation equal to zero because the noise components are not considered at the transmitter side, so:

$$
\mathcal{R}_{R R}=\bar{G}_{8 \times 8} \mathcal{R}_{X_{R F} X_{R F}} \bar{G}_{8 \times 8}{ }^{H}+\sigma_{n}^{2} I
$$

Where, $n_{R F} n_{R F}{ }^{H}=\sigma_{n}^{2}$

$$
\mathcal{R}_{R R}=P_{t} \bar{G}_{8 \times 8} \bar{G}_{8 \times 8}{ }^{H}+\sigma_{n}^{2} I
$$

The above formula represents the covariance matrix of received symbol vector $\bar{R}$.

In a similar manner:

$$
\begin{aligned}
& \mathcal{R}_{R X_{R F}}=E\left\{\bar{R} X_{R F}\right\}=E\left\{\left(G_{8 \times 8} \bar{X}_{R F}+\right.\right. \\
& \left.\left.\bar{n}_{R F}\right) \bar{X}_{R F}{ }^{H} \bar{R}\right\}=E\left\{\bar{G}_{8 \times 8} \bar{X}_{R F} \bar{X}_{R F}{ }^{H}+\right. \\
& \left.\bar{n}_{R F} \bar{X}_{R F}\right\}=P_{t} \bar{G}_{8 \times 8}
\end{aligned}
$$

The second term also tends to zero for the same reason mentioned previously.

However,

$$
\begin{aligned}
& \bar{L}=\mathcal{R}_{R R}{ }^{-1} \mathcal{R}_{R X_{R F}}=\left(P_{t} \bar{G}_{8 \times 8} \bar{G}_{8 \times 8}{ }^{H}+\right. \\
& \left.\sigma_{n}^{2} I\right)^{-1}\left(P_{t} \bar{G}_{8 \times 8}\right)=\left(P_{t} \bar{G}_{8 \times 8} \bar{G}_{8 \times 8}{ }^{H}+\right. \\
& \left.\sigma_{n}^{2} I\right)^{-1} \bar{G}_{8 \times 8}
\end{aligned}
$$

This is the LMMSE Estimator for $8 \times 8$ MIMO channel. And the estimated signal for $8 \times 8$ MIMO:

$$
\begin{aligned}
& \hat{X}_{R F}=\bar{L}^{H} \bar{R} \\
& \hat{X}_{R F}=P_{t} \bar{G}_{8 \times 8}{ }^{H}\left(\bar{G}_{8 \times 8} \bar{G}_{8 \times 8}{ }^{H}+\sigma_{n}^{2} I\right)^{-1} \bar{R}
\end{aligned}
$$

Now,

$$
\begin{aligned}
& \bar{G}_{8 \times 8}{ }^{H}\left(P_{t} \bar{G}_{8 \times 8} \bar{G}_{8 \times 8}{ }^{H}+\sigma_{n}^{2} I\right)^{-1}= \\
& P_{t} \bar{G}_{8 \times 8} \bar{G}_{8 \times 8}{ }^{H}+ \\
& \sigma_{n}^{2} \bar{G}_{8 \times 8}{ }^{H}={ }^{H} P_{t} \bar{G}_{8 \times 8} \bar{G}_{8 \times 8} \bar{G}_{8 \times 8}{ }^{H}+ \\
& \sigma_{n}^{2} \bar{G}_{8 \times 8}{ }^{H}=P_{t} \bar{G}_{8 \times 8}{ }^{H}{ }_{\left(P_{t} \bar{G}_{8 \times 8} \bar{G}_{8 \times 8}{ }^{H}+\right.}+ \\
& \left.\sigma_{n}^{2} I\right)^{-1} \bar{R}
\end{aligned}
$$

Finally,

$$
\begin{aligned}
& \widehat{X}_{R F_{M M S E}}=P_{t}\left(P_{t} \bar{G}_{8 \times 8}{ }^{H} \bar{G}_{8 \times 8}+\right. \\
& \left.\sigma_{n}^{2} I\right)^{-1} \bar{G}_{8 \times 8}{ }^{H} \bar{R}
\end{aligned}
$$

\section{Results and discussion}

First, Fig. 4 ( $a$ and b) shows the Probability Density Function (PDF) of an irradiance fluctuation and a set of analysis examined the impact of scintillation for FSO link assuming wavelength of $1550 \mathrm{~nm}$, diameter of the receiver collecting lens aperture $\mathrm{D}=0.02$, index of refraction structure parameter $C_{n}^{2}=10^{-18} \quad$ (weak), $C_{n}^{2}=10^{-15}$ (medium), $C_{n}^{2}=10^{-12}$ (strong) $\mathrm{m}^{-2 / 3}$ respectively, 
and the path length is $\mathrm{L}=2000 \mathrm{~m}$.

Fig. 5 compares MMSE with ZF channel estimators where the spectral efficiency [bit/Hz/user] is computed versus the number of antennas. Interestingly, the spectral efficiency was observed to increase as the number of antennas are increased in massive MIMO scheme where MMSE gets better spectral efficiency than ZF as the number of antennas are increased.

Fig. 6 compares the BER achieved with LT code considering different massive MIMO schemes (coded channel $(4 \times 4,8 \times 8$ and $16 \times 16)$ and uncoded channel $(16 \times 16))$ for RF link. We observed that the $16 \times 16$ massive MIMO performs better than the other schemes. The adapt of LT code is further improves the BER performance when compared with the same schemes without code. The results, as shown in Fig. 6 indicate that the $16 \times 16$ has a gain of $3.5 \mathrm{~dB}$ as compared with $4 \times 4$ implementing LT code. It is observed that the continuous line graph represents the Bit-Error-Rate (BER) curve for simulated massive MIMO-LT code which is a large

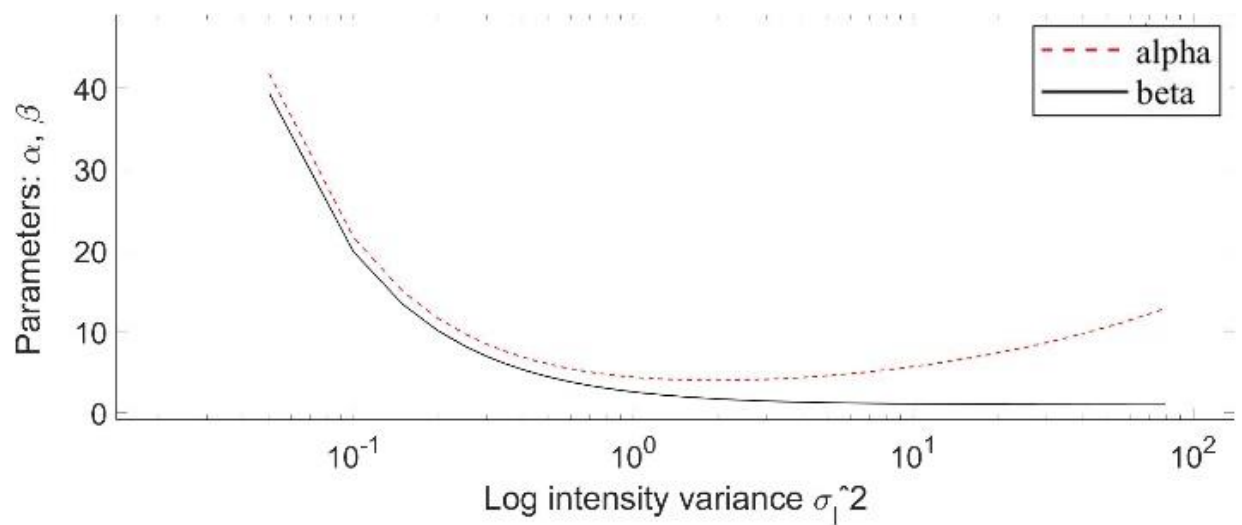

(a)

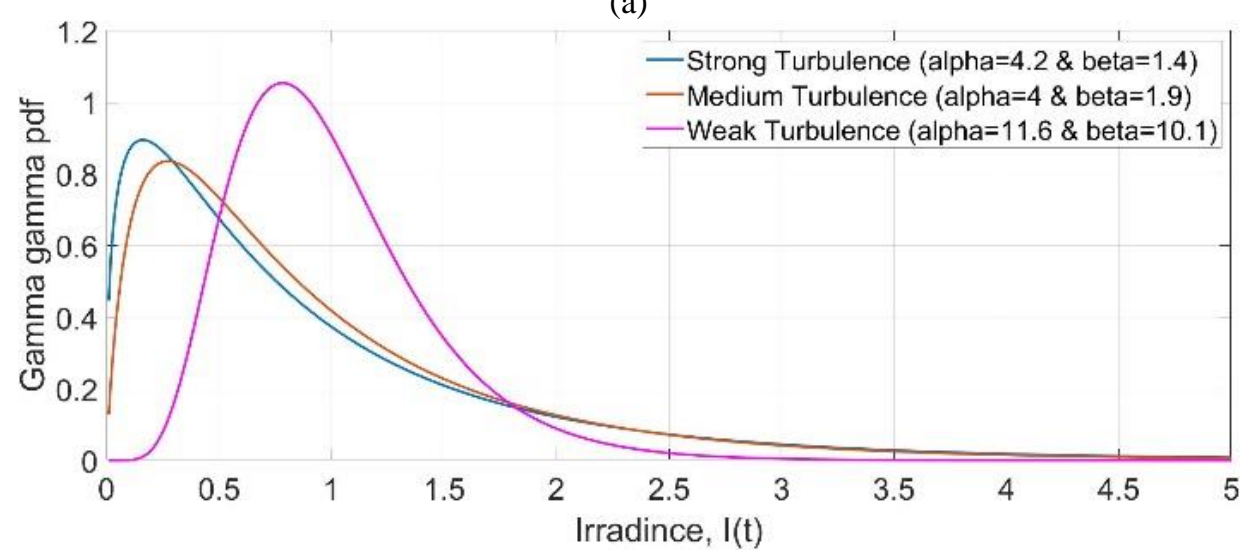

(b)

Figure. 4 PDF of: (a) irradiance fluctuation (alpha and beta) and (b) different turbulence conditions (weak, medium and strong)

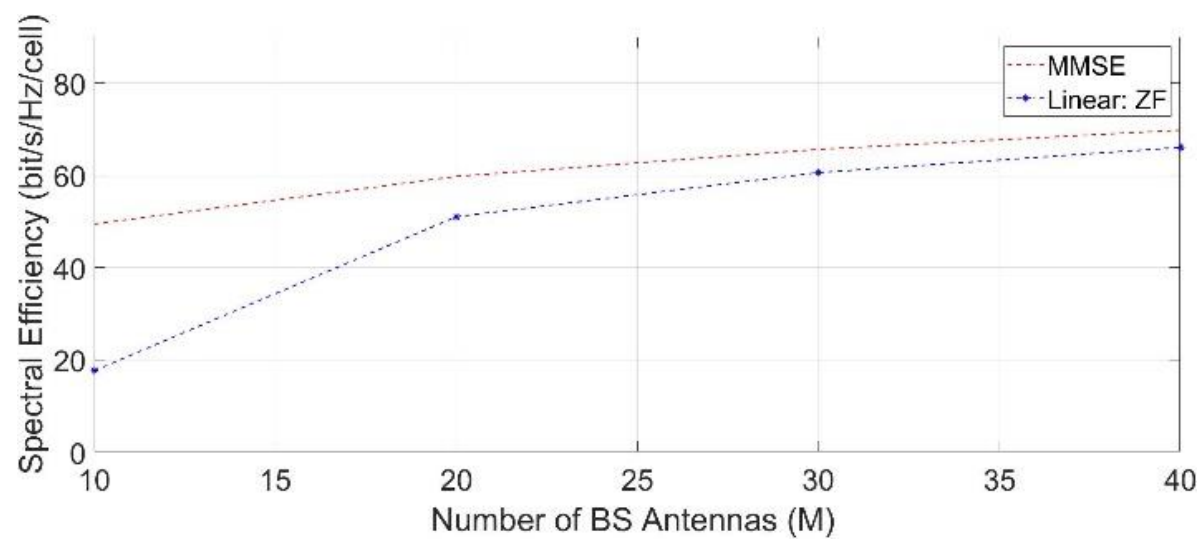

Figure. 5 Spectral efficiency of MMSE and ZF for different number of antennas 


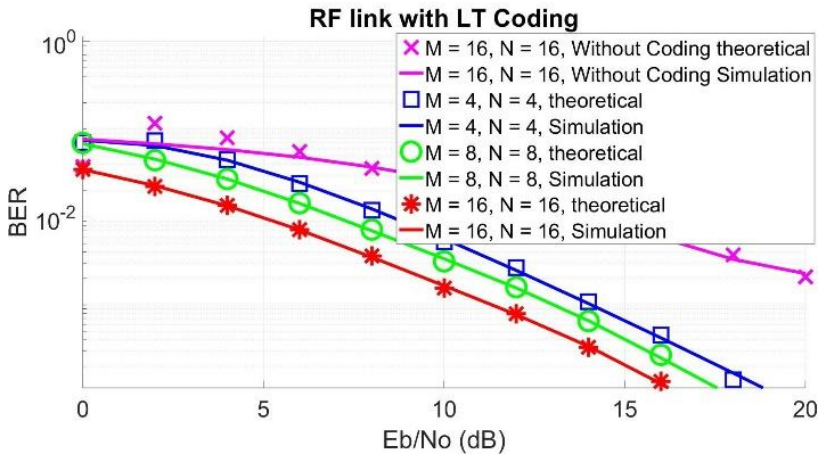

Figure. 6 RF link with LT coding

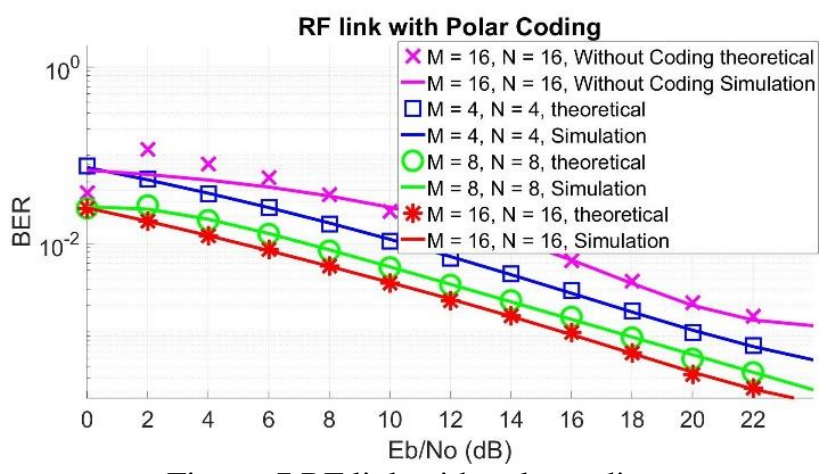

Figure. 7 RF link with polar coding

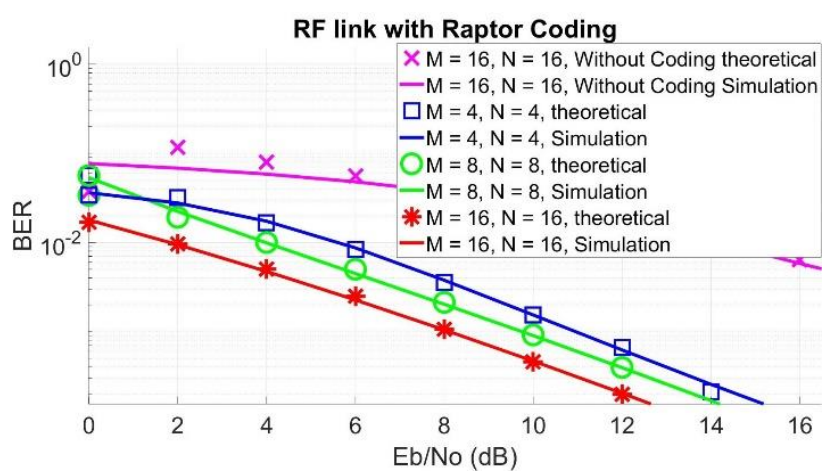

Figure. 8 RF link with raptor coding

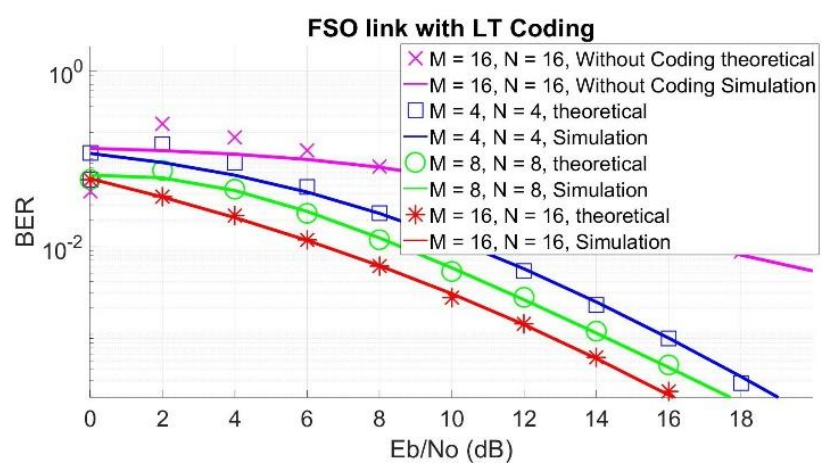

Figure. 9 FSO link with LT coding

extent that aligns with the shaped line (square, circle and star) represented theoretical BER.

Fig. 7 shows the advantage of using massive MIMO schemes implementing a polar code for RF link. With successive increase in the number of transmitting and receiving antennas implementation polar code, $16 \times 16$ improves further the BER as compared with system without implementing polar code. It is observed that $16 \times 16$ implementing polar code has a gain of $8 \mathrm{~dB}$ when compared to $16 \times 16$ massive MIMO with uncoded channel.

Issued related to severe effects of deep fade due to the Mie scattering and other atmospheric effects that causing destructive interference can be reduced Significantly using a raptor code. Fig. 8 presents the use of raptor code in the proposed system. Certainly, $16 \times 16$ MIMO has a superior performance when compared with other MIMO schemes adopted in this paper as well as the system with uncoded channel. As expected, with increasing the transmitting and receiving antennas utilizing raptor code, the BER will degrade at high $\mathrm{E}_{\mathrm{b}} / \mathrm{N}_{\mathrm{o}}$.

Turning now to the simulation of the FSO link considering the specifications reported above.

Fig. 9 compares the different schemes of massive MIMO considering $\mathrm{M}$ emitting diodes at emitter and $\mathrm{N}$ photodetectors at the receiving side. There was a significant improvement in the BER performance when the system implemented LT code. For $16 \times 16$, it requires $12.5 \mathrm{~dB} \mathrm{E}_{b} / \mathrm{N}_{o}$ to achieve BER of $10^{-3}$ while it requires $14 \mathrm{~dB}$ and $16 \mathrm{~dB}$ to achieve same BER for $8 \times 8$ and $4 \times 4$ respectively. In Fig. 9 the continuous line graph represents BER curve for simulated massive MIMO-LT code which is overlapped with a maximum deviation of about 0.5 $\mathrm{dB}$ by the shaped curve (circle, square and star)

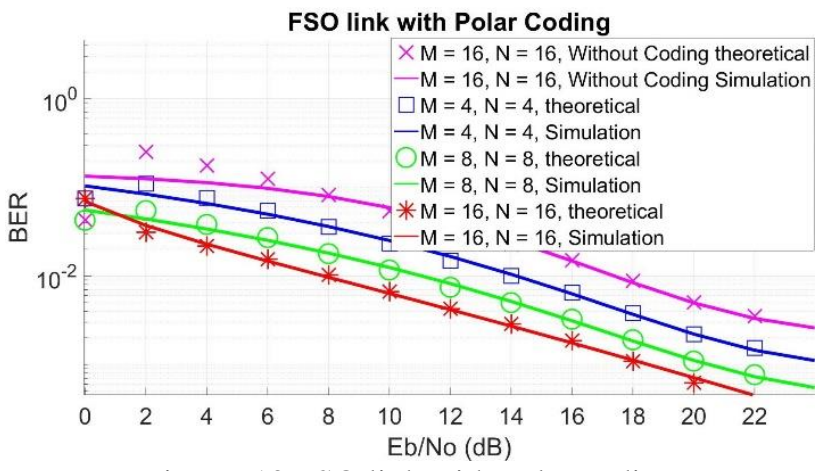

Figure. 10 FSO link with polar coding

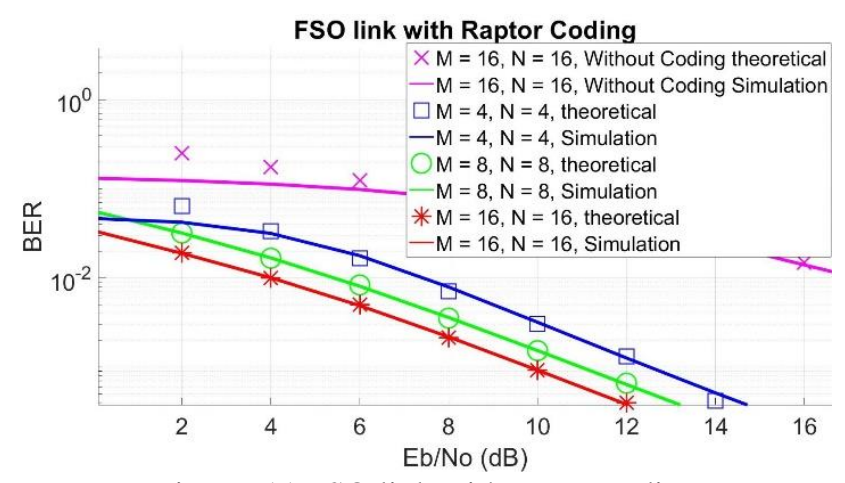

Figure. 11 FSO link with raptor coding 
represented theoretical BER.

When $(4 \times 4,8 \times 8$ and $16 \times 16)$ where simulated with polar code, significant difference in BER was detected between the different MIMO schemes reported above. Uncoded channel gets worse performance as compared with $(4 \times 4,8 \times 8$ and $16 \times 16)$ in Fig. 10.

As shown in Fig. 11, the raptor code reported better performance for $16 \times 16$ than the other two schemes $8 \times 8$ and $4 \times 4$ and uncoded scheme.

One interesting finding is that the Raptor code gets better BER performance whether RF link or FSO link. This is due to the ability of this code to reduce the noise and fading because the structure of this code includes LDPC which reported significantly lower BER and LT code which reported its ability to combat noise and fading.

However, the link availability in different channel conditions can be ensured with the existence of either RF link or FSO link (hybrid system)

Another important finding was that with successive increases in antennas at transmitter and receiver reaching to massive MIMO, the BER performance gets better. Comparing the three channel codes utilized in this paper, it can be seen that the Raptor code gets the best results due to its ability to combat deep fading. As shown in Fig. 6 to Fig. 11, the Raptor code reported a more significant enhancement in BER performance than the other two channel codes (LT and polar)

Overall, these results indicate that using Raptor channel code which has the ability to combat the deep fade with a massive MIMO technique can significantly improve the overall system performance in severe channel conditions.

These results further support the idea of using RF link as a standby backup for FSO link in obtaining a better solution to achieve a suitable data rates in difficult channel conditions.

Fig. 12 provides the results obtained from the hybrid FSO/RF system according to results reported in Fig. 6 to Fig. 11 which indicate that the Raptor

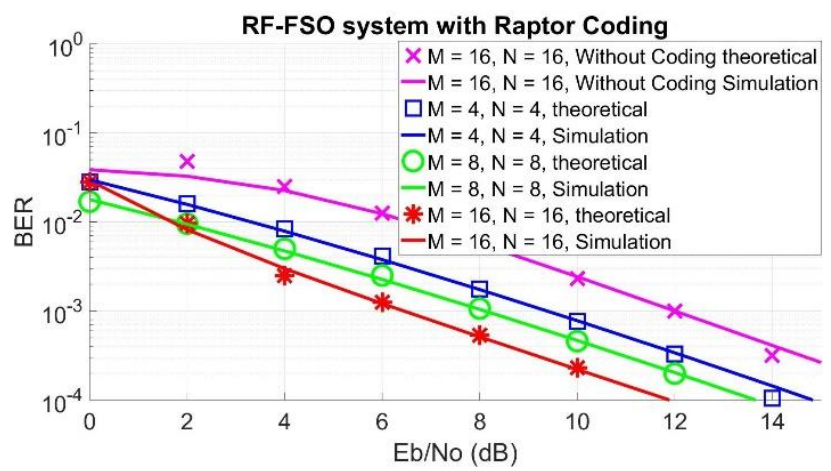

Figure. 12 RF-FSO system with Raptor coding
Table 5. Comparison of the BER achieved with Eb/No $=10 \mathrm{~dB}$ for different links, MIMO schemes and coding techniques

\begin{tabular}{|l|c|c|}
\hline \multicolumn{1}{|c|}{ Channel } & Eb/No $(\mathrm{dB})$ & BER \\
\hline RF 16x16 without code & 10 & 0.0266 \\
\hline RF 4x4 with polar code & 10 & 0.0111 \\
\hline RF 8x8 with polar code & 10 & 0.0054 \\
\hline RF 16x16 with polar code & 10 & 0.0036 \\
\hline RF 4x4 with LT code & 10 & 0.0056 \\
\hline RF 8x8 with LT code & 10 & 0.0033 \\
\hline RF 16x16 with LT code & 10 & 0.0016 \\
\hline RF 4x4 with Raptor code & 10 & 0.0015 \\
\hline RF 8x8 with Raptor code & 10 & 0.0009 \\
\hline RF 16x16 with Raptor code & 10 & 0.0004 \\
\hline FSO 16x16 without code & 10 & 0.0601 \\
\hline FSO 4x4 with polar code & 10 & 0.0250 \\
\hline FSO 8x8 with polar code & 10 & 0.0124 \\
\hline FSO 16x16 with polar code & 10 & 0.0066 \\
\hline FSO 4x4 with LT code & 10 & 0.0122 \\
\hline FSO 8x8 with LT code & 10 & 0.0057 \\
\hline FSO 16x16 with LT code & 10 & 0.0029 \\
\hline FSO 4x4 with Raptor code & 10 & 0.0031 \\
\hline FSO 8x8 with Raptor code & 10 & 0.0015 \\
\hline FSO 16x16 with Raptor code & 10 & 0.0009 \\
\hline FSO/RF 16x16 without code & 10 & 0.0024 \\
\hline FSO/FSO 4x4 with Raptor code & 10 & 0.0007 \\
\hline FSO/FSO 8x8 with Raptor code & 10 & 0.0004 \\
\hline FSO/FSO 16x16 with Raptor code & 10 & 0.0002 \\
\hline
\end{tabular}

code has a superior performance when a massive MIMO is considered. The results show that the $16 \times 16$ gets a better BER performance for the proposed hybrid system adopting Raptor channel code. There was a significant difference between the coded and uncoded channel, coded $16 \times 16$ has a gain of $5.5 \mathrm{~dB}$ as compared with uncoded $16 \times 16$.

Finally, table 5 shows the BER achieved with $\mathrm{Eb} /$ No equals to $10 \mathrm{~dB}$ for different MIMO schemes and different coding techniques. As shown in the table, using Raptor code with the RF link with $8 \times 8$ and 16x16 MIMO schemes and FSO link with 16x16 MIMO scheme can achieve BER near the BER achieved with the FSO/RF link proposed, but with taking into consideration the availability of the system in different weather conditions, FSO/RF link has the better performance.

\section{Conclusions}

In this paper, a hybrid FSO/RF massive MIMO system is presented, in which the different channel codes such as LT, polar and Raptor codes are used. The main contribution of the current study was to determine the BER which suffers inferiority when the link undergo to deep fade. Channel codes used in this paper are capable to mitigate the severe channel conditions due to the atmospheric turbulence as well 
as destructive interference in the $\mathrm{mm}$ wave ranges and in evaluating the maximized SNR using beamforming technique. The research has shown that the hybrid system guarantees reliable system and very high availability in the severe channel conditions. The results of this work show that the spectral efficiency increases with increasing the number of antennas, where the spectral efficiency for MMSE channel estimator is about $58 \mathrm{bit} / \mathrm{s} / \mathrm{Hz} /$ cell while it is $40 \mathrm{bit} / \mathrm{s} / \mathrm{Hz} /$ cell for ZF. BER performance will be improved in the hybrid system with adopting a large number of antennas at transmitter and receiver sides. The theoretical results which get by a derived mathematical model for the proposed system are consistent with the results got by Monte Carlo simulations. Also, the results of this research support the idea that the suitable error correction codes which combat the deep fade can improve the system reliability. Whereas, $16 \times 16$ MIMO implemented Raptor code got better gain about $14 \mathrm{~dB}$ as compared to same system without implementing Raptor code.

\section{Conflicts of interest}

The authors declare no conflict of interest.

\section{Author contributions}

Conceptualization, L. Abdulameer; software, T. Abdulabbas; formal analysis, L. Abdulameer; writing - original draft preparation, review and editing, L. Abdulameer, and T. Abdulabbas; supervision, L. Abdulameer.

\section{References}

[1] B. Makki, T. Svensson, M. B. Pearce, and M. S. Alouini, "On the performance of millimeter wave-based RF-FSO multi-hop and mesh networks", IEEE Trans. Wirel. Commun., Vol. 16, No. 12, pp. 7746-7759, 2017.

[2] A. Tahami, A. Dargahi, K. Abedi, and A. C. Motlagh, "A new relay based architecture in hybrid RF/FSO system", Phys. Commun., Vol. 36, 2019.

[3] M. A. Amirabadi and V. T. Vakili, "A novel hybrid FSO/RF communication system with receive diversity", Optik (Stuttg)., Vol. 184, pp. 293-298, 2019.

[4] A. Abdulhussein, A. Oka, T. Nguyen, and L. Lampe, "Rateless coding for hybrid free-space optical and radio-frequency communication", IEEE Trans. Wirel. Commun., Vol. 9, No. 3, pp. 907-913, 2010.

[5] S. Bloom and W. Hartley, "The last-mile solution: hybrid FSO radio", AirFiber Inc., 2002.
[6] M. Siddharth, S. Shah, and R. Swaminathan, "Outage analysis of adaptive combining scheme for hybrid FSO/RF communication", In: Proc. of 2020 Natl. Conf. on Commun.(NCC), 2020.

[7] S. Vangala and H. P. Nik, "Optimal hybrid RFwireless optical communication for maximum efficiency and reliability", In: Proc. of 200741 st Annual Conference on Information Sciences and Systems, pp. 684-689, 2007.

[8] S. Vangala and H. P. Nik, "A highly reliable FSO/RF communication system using efficient codes", In: Proc. of IEEE GLOBECOM 2007 IEEE Glob. Telecommun. Conf., pp. 2232-2236, 2007.

[9] L. Han, H. Jiang, Y. You, and Z. Ghassemlooy, "On the performance of a mixed RF/MIMO FSO variable gain dual-hop transmission system", Opt. Commun., vol. 420, pp. 59-64, 2018.

[10] B. Bag, A. Das, C. Bose, and A. Chandra, "Link Quality of a RF-assisted Relayed FSO System under Turbulence, Pointing Error and Fading", In: Proc. of 2020 Int. Conf. Comput. Electr. Commun. Eng. (ICCECE), pp. 1-6, 2020.

[11] L. Chen and W. Wang, "Multi-diversity combining and selection for relay-assisted mixed RF/FSO system", Opt. Commun., Vol. 405, pp. 1-7, 2017.

[12] H. Liang, Y. Li, M. Miao, C. Gao, and X. Li, "Unified performance analysis of mimo mixed rf/fso relaying system”, Appl. Sci., Vol. 11, No. 7, 2021.

[13] D. R. Zaghar, H. N. Abdullah, and I. M. Farhan, "Performance analysis of systematic raptor codes in low power regime", Int. J. Intell. Eng. Syst., Vol. 13, No. 4, pp. 283-292, 2020.

[14] Z. Wei, B. Li, W. Hu, W. Guo, and C. Zhao, "Hamming-Luby rateless codes for molecular erasure channels", Nano Commununication Networks, vol. 23, p. 100280, 2020.

[15] C. Wang, L. Suo, D. Bian, J. Lv, and S. Tian, "Performance Analysis and Design of LT Codes Under the Gaussian Elimination Algorithm in Wireless Sensor Networks", IEEE Access, Vol. 7, pp. 55797-55806, 2019. 